Aline Albuquerque ${ }^{1}$

\title{
A SEGURANÇA DO PACIENTE À LUZ DO REFERENCIAL DOS DIREITOS HUMANOS
}

Patient safety in light of the human rights framework

${ }^{1}$ University of Essex. Colchester, Inglaterra.

Correspondência: Aline Albuquerque. E-mail: alineaoliveira@hotmail.com.

Recebido em: 30/08/2015. Revisado em: 18/11/2015. Aprovado em: 07/12/2015. 


\section{RESUMO}

Este artigo tem por objetivo a elaboração de aporte teórico que ampare a concepção de que a segurança do paciente se interconecta com a obrigação dos Estados de preservar a vida de seus jurisdicionados, bem como é uma expressão do direito à saúde sob o prisma do Direito Internacional dos Direitos Humanos - que compreende os tratados de direitos humanos e a jurisprudência internacional emanada dos órgãos de direitos humanos situados na ONU, no Sistema Interamericano de Direitos Humanos e no Sistema Europeu de Direitos Humanos. Com o escopo de desenvolver a acepção de segurança do paciente sustentada sob o prisma do direito à vida e do direito à saúde, adotou-se pesquisa de revisão bibliográfica e documental, destacando-se o levantamento e a análise de relatórios e decisões produzidos pelos órgãos de direitos humanos apontados. Em conclusão, o direito ao cuidado em saúde seguro, derivado do direito à vida e do direito à saúde, deve ser objeto de regulação por parte dos Estados. Ou seja, sustenta-se neste artigo que certo elenco de medidas gerais de segurança do paciente seja legalmente instituído, de modo a enunciar explicitamente o direito ao cuidado em saúde seguro, enquanto um dos direitos humanos do paciente. Com efeito, a implantação da cultura de segurança do paciente é uma obrigação dos Estados; sua negligência em efetivá-la por meio de medidas legislativas, de políticas públicas e de orçamento próprio consiste em infringência aos comandos internacionais de direitos humanos.

\section{Palavras-Chave}

Direito à Saúde; Direito à Vida; Direitos Humanos; Segurança do Paciente.

\section{ABSTRACT}

This article aims to draw up a theoretical contribution to sustain the view that patient safety is interconnected with the obligation of States to preserve the lives of their citizens and that patient safety is an expression of the right to health, from the perspective of international human rights, comprising the human rights treaties and international jurisprudence emanating from human rights bodies located at the United Nations, the Inter-American Human Rights System and the European Human Rights System. With the aim of developing the patient safety based on the right to life and the right to health, we have adopted a bibliographical and a documentary research, highlighting the review and analysis of reports and decisions made by human rights organs. In conclusion, the right to safe healthcare, derived from the right to life and the right to health, should be subject to state regulations, that is, we argue that the state should establish a certain cast of general measures of patient safety, in order to explicit the right to safe healthcare, as one of the human rights of the patient. Indeed, the implementation of a patient safety culture is a State obligation; its disregard in defining it as a result of a legislative, public policy or budget lack, involves an international human rights violation.

\section{Keywords}

Human Rights; Patient Safety; Right to Health; Right to Life. 


\section{Introdução}

A segurança do paciente é uma questão de saúde pública de alcance global, cujo escopo central consiste na redução do "risco de danos desnecessários associa-

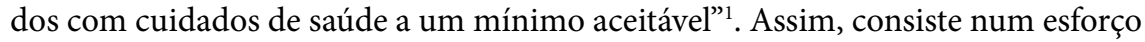
concertado para prevenir ações inseguras na ambiência dos sistemas de saúde.

Países de alta, média e baixa renda contam atualmente com políticas e programas a fim de reduzir o risco de danos evitáveis associados a cuidados de saúde a um mínimo aceitável ${ }^{2}$, embora seja recente o reconhecimento de que a segurança do paciente é um grave problema de saúde pública mundial.

A partir do desenvolvimento tecnológico da medicina, foram criados riscos adicionais para a saúde humana, e os cuidados em saúde incrementam o risco de dano ao paciente. Um dos primeiros estudos sobre a temática é o relatório intitulado "Errar é Humano", publicado em 1999 nos Estados Unidos. O relatório enunciou estratégias amplas dirigidas a governos, provedores de serviços, profissionais de saúde e pacientes com o objetivo de reduzir a ocorrência de eventos adversos ${ }^{3}$ nos cuidados em saúde, promovendo uma cultura baseada na abordagem sistêmica do erro não focada no erro individual - ou seja, sua perspectiva aparta-se da responsabilização individual e isolada do profissional de saúde ${ }^{4}$.

Durante os últimos dez anos, a segurança do paciente tem sido cada vez mais considerada como um tema de alcance global, mas ainda há um longo caminho a ser percorrido. Segundo a Organização Mundial da Saúde (OMS), nos países ricos, "um em cada 10 pacientes sofre algum tipo de dano durante seu período de permanência no hospital"5. Na OMS há o reconhecimento, que cresce gradualmente, de que a segurança do paciente é uma dimensão fundamental da cobertura universal de saúde ${ }^{6} \mathrm{Na} 55^{\mathrm{a}}$ Assembleia Mundial da Saúde, os Estados-Membros adotaram em maio de 2002 a Resolução WHA55.18, em que se comprometem "a prestar a

${ }^{1}$ ORGANIZACIÓN MUNDIAL DE LA SALUD - OMS. Marco Conceptual de la Clasificación Internacional para la Seguridad del Paciente. Versión 1.1. 2009. p. 16. Disponível em: <http://www.who.int/patientsafety/ implementation/icps/icps_full_report_es.pdf>. Acesso em: 28 out. 2014.

${ }^{2}$ REMON, C.A. OMS. Seguridad del paciente: curso virtual de introducción a la investigación en seguridad del paciente. OMS. Genebra: OMS, 2012. 480 min. Disponível em: <http://www.who.int/patientsafety/ research/curso_virtual/es/>. Acesso em: 6 out. 2016.

${ }^{3}$ Segundo Walter Mendes, há 24 definições de erro e 14 de evento adverso. 0 autor também define o erro como o evento não intencional que resultou ou poderia ter resultado em dano desnecessário ao paciente. Neste artigo serão usados indistintamente os termos "evento adverso" e "erro", sem levar em conta a intencionalidade do ato. MENDES, Walter. Segurança do paciente. Disponível em: <http://portal.anvisa.gov.br/wps/wcm/connect/8 adff400417e01dd8ec6ee22d1e56fc9/Walter_Mendes.pdf?MOD=AJPERES>. Acesso em: 10 nov. 2015.

${ }^{4}$ NATIONAL ACADEMY OF SCIENCES. Institute of Medicine: shaping the future for health. Disponivel em: <https://www.iom.edu/ /media/Files/Report\%20Files/1999/To-Err-is-Human/To\%20Err\%20is\%20 Human\%201999\%20\%20report\%20brief.pdf. Acesso em: 28 out. 2014.

${ }^{5}$ ORGANIZACIÓN MUNDIAL DE LA SALUD - OMS. Marco Conceptual de la Clasificación Internacional para la Seguridad del Paciente, cit., p. 16.

${ }^{6}$ ORGANIZACIÓN MUNDIAL DE LA SALUD - OMS. 10 datos sobre seguridad del paciente. Disponível em: <http:// www.who.int/features/factfiles/patient_safety/patient_safety_facts/es/>. Acesso em: 30 jul. 2014. 
maior atenção possível ao problema da segurança do paciente e a estabelecer e consolidar os sistemas de base científica necessários para melhorar a segurança e a qualidade dos cuidados básicos de saúde"7. No mesmo sentido, a Assembleia deliberou sobre a necessidade de a OMS elaborar normas e padrões globais, bem como de os Estados-Membros se esforçarem para adotar políticas e ações relacionadas à segurança do paciente ${ }^{8}$. De fato, a Assembleia Geral da OMS instou os Estados-Membros com vistas a chamar sua atenção para a segurança do paciente. Sendo assim, verifica-se que a adoção da resolução citada sublinhou a importância da segurança do paciente como um problema de saúde global.

No Brasil, o Programa Nacional de Segurança do Paciente foi instituído pelo Ministério da Saúde em abril de 2013, "com o objetivo geral de contribuir para a qualificação do cuidado em saúde, em todos os estabelecimentos de Saúde do território nacional, quer públicos, quer privados"’. A instituição do Programa reflete o movimento mundial na direção da assunção por parte dos Estados de sua obrigação de proteger os pacientes dos danos decorrentes dos cuidados em saúde e de prover o acesso a bens e serviços de saúde de qualidade.

Constata-se, desse modo, que o tema da segurança do paciente foi comumente abordado, seja no plano internacional seja no nacional, sob a perspectiva da saúde. Sendo assim, os estudos e pesquisas na área dos direitos humanos sobre segurança do paciente são escassos. Em contrapartida, encontram-se em maior número investigações que entrelaçam o campo jurídico com a segurança do paciente, enfatizando os direitos dos pacientes enquanto consumidor. A perspectiva do paciente enquanto titular de direitos humanos no contexto da segurança é inovadora. Desse modo, esta pesquisa parte do pressuposto de que a separação entre a segurança do paciente e o referencial teórico-normativo dos direitos humanos deu-se em função de ter sido construída por profissionais da saúde não familiarizados com a linguagem dos direitos humanos. Por outro lado, os estudiosos de direitos humanos, especificamente do direito à saúde, ainda não se dedicaram suficientemente à investigação da segurança do paciente, enquanto campo conectado com o direito à saúde, integrante da qualidade e elemento do direito apontado. Portanto, registra-se a exiguidade da reflexão sobre segurança do paciente sob o viés dos direitos humanos.

A escolha do referencial teórico-normativo dos direitos humanos ancora-se na concepção de que tais direitos são essenciais para a proteção dos pacientes no

${ }^{7}$ WORLD HEALTH ORGANIZATION - WHO. Resolution WHA55.18. Disponivel em: <http://apps.who.int/gb/ archive/pdf_files/WHA55/ewha5518.pdf?ua=1>. Acesso em: 08 set. 2016.

${ }^{8}$ ORGANIZACIÓN MUNDIAL DE LA SALUD - OMS. Marco Conceptual de la Clasificación Internacional para la Seguridad del Paciente, cit.

${ }^{9}$ MINISTÉRIO DA SAÚDE; FUNDAÇÃO OSWALDO CRUZ; AGÊNCIA NACIONAL DE VIGILÂNCIA SANITÁRIA. Documento de referência para o Programa Nacional de Segurança do Paciente. Disponível em: <http:// bvsms.saude.gov.br/bvs/publicacoes/documento_referencia_programa_nacional_seguranca.pdf $>$ Acesso em: 20 ago. 2015 
contexto dos cuidados de saúde, porquanto impõe aos Estados a obrigação de adotar medidas concretas com vistas a prover serviços e bens de saúde de qualidade - e, em consequência, com segurança. Sendo assim, esta pesquisa tem como escopo demonstrar e desenvolver as seguintes concepções: (a) a segurança do paciente é uma expressão concreta do direito à vida, logo as políticas de segurança do paciente são modos de realização de tal direito; $\mathrm{e}(\mathrm{b})$ a segurança do paciente integra a qualidade, elemento do direito de toda pessoa de desfrutar o mais elevado nível possível de saúde física e mental ${ }^{10}$.

Sob a perspectiva do direito à vida, eventos adversos decorrentes do processo terapêutico podem acarretar a morte do paciente. Nesse sentido, órgãos internacionais de direitos humanos se posicionaram quanto à interface entre a violação ao direito à vida e a morte de pacientes resultante de erros cometidos por profissionais de saúde. No que toca ao direito à saúde, conforme o arcabouço teórico-normativo desenvolvido no âmbito da Organização das Nações Unidas (ONU) acerca de seu conteúdo, tal direito é constituído por quatro elementos: disponibilidade, acessibilidade, aceitabilidade e qualidade. Nesta pesquisa enfoca-se o último elemento, na medida em que a segurança do paciente é um dos atributos da qualidade.

A fim de demarcar a abordagem teórica deste artigo, o referencial do direito à vida e do direito à saúde ora empregado fundamenta-se na jurisprudência internacional, que é construída pelos relatórios, diretrizes, princípios, recomendações e tratados produzidos pelos órgãos internacionais de direitos humanos, notadamente integrantes do Sistema de Direitos Humanos das ONU, do Sistema Europeu de Direitos Humanos e do Sistema Interamericano de Direitos Humanos. Os sistemas ONU e Interamericano foram escolhidos em razão de os pacientes no Brasil poderem recorrer a eles. Quanto ao Europeu, embora o Brasil não se submeta a seus órgãos de monitoramento, é o sistema mais avançado em termos de direitos humanos dos pacientes, contando com substantiva jurisprudência sobre cuidados em saúde, bioética e consentimento informado, por exemplo - por isso é indispensável seu exame.

No que tange aos passos metodológicos, adotou-se a pesquisa de revisão bibliográfica e documental, destacando-se o levantamento e a análise de artigos e obras concernentes à segurança do paciente, bem como relatórios e decisões produzidos pelos órgãos internacionais apontados quanto ao referencial teórico-normativo dos direitos humanos. Em consonância com tais passos, estruturou-se este artigo em duas partes: a primeira versa sobre o direito à vida; a segunda, acerca do direito à saúde; e ambas, sobre as respectivas conexões com a segurança do paciente. A seguir, passa-se à aplicação do direito à vida ao tema da segurança do paciente.

\footnotetext{
${ }^{10}$ Denominado neste artigo como direito à saúde.
} 


\section{A segurança do paciente e sua interconexão com o direito à vida}

\section{Demarcação substantiva do direito à vida na esfera dos cuidados em saúde}

O paciente, como qualquer pessoa humana, possui o direito primordial à vida. Ao paciente são aplicados todos os dispositivos legais sobre o direito à vida visando a protegê-lo e a punir os que atentem contra sua vida. O direito à vida é um pré-requisito para o desfrute dos demais direitos, logo, caso não seja respeitado, todos "os demais direitos humanos carecem de sentido"11. Na esfera dos cuidados em saúde, indagam-se quais seriam as situações específicas concernentes ao direito à vida do paciente. Em primeiro lugar, se pensaria nas questões atinentes à terminalidade da vida - por exemplo, à eutanásia e ao suicídio assistido. Contudo, seguindo a jurisprudência internacional, esses temas são tratados no âmbito do direito à privacidade, pois se nota que o reconhecimento dos direitos dos pacientes em situações de terminalidade da vida não se fundamenta no direito à vida, mas sim no direito de conduzir sua própria vida conforme suas escolhas e, portanto, de acordo com o direito à privacidade. Ilustrando: para a Corte Europeia de Direitos Humanos, do direito à vida não se infere para o paciente o direito de morrer ${ }^{12}$, na medida em que o direito à vida não é tão extensivo de modo que abarque o direito do paciente de manejar sua própria morte ${ }^{13}$.

O direito à vida, além de ensejar a obrigação estatal de respeitar, ou seja, de se abster de suprimir a vida de alguém, também impele os Estados a prover condições de vida digna ${ }^{14}$ adotando medidas positivas, como as direcionadas a diminuir a mortalidade infantil e a aumentar a esperança de vida das populações ${ }^{15}$. O direito à vida enseja basicamente três tipos de obrigações para os Estados: (a) o dever de se abster de retirar a vida de alguém, salvo nas exceções legalmente previstas, tais como a legítima defesa; (b) o dever de investigar as mortes suspeitas e de punir os culpados, conforme o devido processo legal e os remédios judiciais cabíveis; e (c) o dever legal de adotar medidas positivas que previnam a morte evitável. Assim, os Estados devem não apenas preocupar-se com as mortes resultantes do uso ilegal da força por parte de seus agentes, mas também devem tomar providências para salvaguardar a vida das pessoas em seu território ${ }^{16}$. Essa obrigação é manifesta em situações em que há

\footnotetext{
${ }^{11}$ Corte Interamericana de Direitos Humanos. Caso de los "Niños de la Calle" (Villagrán Morales y otros) Vs. Guatemala. Sentencia de 19 de noviembre 1999. (Fondo). p. 40.

${ }^{12}$ ECHR. Pretty v. the United Kingdom, no. 2346/02, judgment of 29 April 2002.

${ }^{13}$ HERRING, J. Medical law. London: Pearson, 2013.

${ }^{14}$ BELETSKY, L.; EZER, T.; OVERALL, J.; BYRNE, I.; COHEN, J. Advancing human rights in patient care: the law in seven transitional countries. Disponível em: <http://www.opensocietyfoundations.org/sites/default/files/ Advancing-Human-Rights-in-Patient-Care-20130516.pdf>. Acesso em: 28 jan. 2015.

${ }^{15}$ COMISIÓN DE DERECHOS HUMANOS. Recopilación de las Observaciones Generales Adoptadas por el Comité de Derechos Humanos de Naciones Unidas. № 6 - Derecho a la vida (art. 6). Disponível em: <http:// www.derechos.org.ve/pw/wp-content/uploads/CDH1.pdf>. Acesso em: 8 fev. 2015.

${ }^{16}$ ECHR.Case of Belenko V. Russia. Judgment.18 December 2014.
} 
real risco de morte ${ }^{17}$. Nessa linha, a Comissão Interamericana de Direitos Humanos assentou que o direito à vida abarca o acesso a medicamentos antirretrovirais para tratamento de HIV/AIDS, pois há risco iminente de morte caso o tratamento não seja provido pelo Estado ${ }^{18}$.

No item seguinte será desenvolvida a acepção da segurança do paciente enquanto expressão do direito à vida com base na jurisprudência internacional.

\section{A segurança do paciente enquanto expressão do direito à vida}

A segurança do paciente deve ser uma das suas principais preocupações, assim como a de seus familiares e dos profissionais de saúde ${ }^{19}$, pois há que se impedir que danos evitáveis ocorram ao paciente, principalmente a sua morte, em decorrência dos cuidados em saúde ${ }^{20}$. Por exemplo, chama-se atenção para o caso de dois recém-nascidos internados no Cedars-Sinai Medical Center, hospital situado na cidade de Los Angeles, Estados Unidos, cujas mortes resultaram de superdose de heparina; e o caso de uma adolescente de 17 anos paciente do Duke University Medical Center, localizado em Durham, Estados Unidos, que morreu ao receber um coração transplantado cujo tipo sanguíneo era incompatível ${ }^{21}$. Esses exemplos evidenciam a urgência de se conceber a segurança do paciente enquanto uma política pública assecuratória de seu direito.

A segurança do paciente é um campo do saber e uma prática novos, sendo definida como "redução, ao mínimo aceitável, do risco de dano desnecessário associado ao cuidado de saúde" 22 . As primeiras iniciativas nessa seara deram-se na Austrália em 1987, mediante a criação da Fundação Australiana de Segurança do Paciente ${ }^{23}$. Um documento notoriamente precursor das discussões sobre o tema - o relatório Errar é Humano, elaborado em 1999 pelo Instituto de Medicina dos Estados Unidos - lançou luz sobre a segurança do paciente enquanto uma das dimensões da qualidade em saúde. De acordo com o relatório, as mortes decorrentes de eventos adversos evitáveis em saúde superavam as provenientes de acidentes automobilísticos,

\footnotetext{
${ }^{17}$ WICKS, Elizabeth. Human rights and healthcare. London: Hart, 2007.

${ }^{18}$ Report No 32/05. Petition 642/03. Admissibility. Luis Rolando Cuscul Pivaral Et Al. (Persons Living With HIV/AIDS). Guatemala. March 7, 2005.

${ }^{19}$ ANNAS, George. The rights of patients. 3. ed. New York University Press, 2004.

${ }^{20}$ Importa ressaltar que as situações de má conduta profissional são enquadradas como "comportamento de imprudente", que significa "atuar com desprezo consciente diante de riscos substanciais e injustificáveis", diferenciando-se do "erro humano" ou do "comportamento de risco", os quais são tratados a partir da perspectiva da segurança do paciente. Essa distinção é muito limítrofe, mas vem sendo adotada na literatura especializada sobre o tema. WACHTER, Robert M. Personal accountability in healthcare: searching for the right balance. In: THE HEALTH Foundation, 2012. Traduzido pelo Proqualis - Instituto de Comunicação e Informação Científica e Tecnológica em Saúde; Fiocruz, 2013.

${ }^{21}$ WACHTER, Robert M. Understanding patient safety. São Francisco: McGraw Hill, 2012.

${ }^{22} \mathrm{OMS}$ apud MENDES, Walter. Taxonomia em segurança do paciente. In: SOUSA, Paulo; Mendes, Walter (Orgs.). Segurança do paciente: conhecendo os riscos nas organizações de saúde. Rio de Janeiro: FIOCRUZ, 2013. p. 60.

${ }^{23}$ CURRIE, L; WATT, S. An introduction to patient safety. In: CURRIE, Lynne (Ed.). Understanding patient safety. London: Quay Books, 2007. p. 11-26.
} 
do câncer de mama e da AIDS. No mesmo estudo, estimou-se a ocorrência anual de 44 mil a 98 mil óbitos evitáveis decorrentes de erros nos cuidados em saúde em hospitais do Estado de Nova Iorque, Estados Unidos ${ }^{24}$. No Estudo Ibero-Americano de Eventos Adversos na Atenção, realizado em cinco países da América Latina, entre 2007 e 2009, demonstrou-se que 10,5\% dos pacientes hospitalizados sofreram algum tipo de evento adverso (EA), e destes, $58,9 \%$ poderiam ter sido evitados ${ }^{25}$.

No plano internacional, a OMS lançou em 2004 seu Programa de Segurança do Paciente, em cumprimento a Resolução WHA55.18, adotada por sua Assembleia Geral que clamava pela atenção da OMS e de seus Estados-Membros para o problema da segurança do paciente, considerado uma questão global de saúde ${ }^{26}$. Assim, a Aliança Mundial para a Segurança do Paciente foi instituída em 2004 com o objetivo de sistematizar conceitos sobre a temática e de propor medidas para reduzir os erros. No âmbito da Aliança Mundial, foram lançados Desafios Globais com o propósito de promover medidas para prevenção de erros. Ainda, criou-se o projeto Patients for Patient Safety (Pacientes pela Segurança do Paciente), que atua globalmente em conjunto com a rede PFPS Champions27. Apesar dos esforços da OMS e dos Estados, o progresso almejado na prevenção de mortes de pacientes decorrentes de eventos adversos ocorridos em seu tratamento não tem sido logrado. Em relatório de pesquisa do ano de 2013 realizado em mais de 30 mil hospitais da Europa, consta que um em cada 18 pacientes apresenta infecção associada aos cuidados em saúde ${ }^{28}$.

O paciente tem o direito de receber cuidados em saúde que sejam seguros, consistindo num desdobramento do direito à vida, na medida em que a ausência de segurança pode, por óbvio, causar a morte do paciente. Os Estados têm a obrigação de respeitar, proteger e realizar o direito à vida e, sendo assim, os agentes governamentais têm o ônus da prevenção - ou seja, são obrigados a prevenir que profissionais e provedores de saúde causem algum dano ao paciente e, em muitas situações, sua morte. Sendo assim, cabe aos Estados adotar medidas legislativas - como leis estabelecendo direitos e deveres na esfera da segurança do paciente - e administrativas - como políticas, programas e campanhas com o fito de instituir ações de segurança do paciente nas unidades de saúde. No mesmo sentido, estratégias de capacitação e formação devem ser fomentadas a fim de que o tema da segurança do paciente faça parte dos planos de ensino dos cursos de graduação na área da saúde e para que profissionais de saúde recebam capacitação acerca da temática ${ }^{29}$.

\footnotetext{
${ }^{24}$ INSTITUTE OF MEDICINE. To err is human: building a safer health system. Washington: National Academy Press, 2000.

${ }^{25}$ ANVISA. Assistência segura: uma reflexão teórica aplicada à prática. Brasília: Anvisa, 2013. Brasília: Anvisa, 2013.

${ }^{26}$ WORLD HEALTH ORGANIZATION - WHO. Resolution WHA55.18, cit.

${ }^{27}$ ORGANIZACIÓN MUNDIAL DE LA SALUD - OMS. 10 datos sobre seguridad del paciente, cit.

${ }^{28}$ FARIA, P.L.; MOREIRA, P.S.; PINTO, L.S. O direito e a segurança do paciente. In: SOUSA, Paulo; MENDES, W. Segurança do paciente: conhecendo os riscos nas organizações de saúde. Rio de Janeiro: FIOCRUZ, 2013. p. 115-134.

${ }^{29} \mathrm{COHEN}$, J.; EZER, T. Human rights in patient care: a theoretical and practical framework. Health and Human Rights, v.15, n. 2, p. 7-19, Dec. 2013.
} 
No âmbito do Sistema Interamericano de Direitos Humanos, o Caso Clínica Pediátrica da Região dos Lagos (Cabo Frio/RJ), em trâmite na Comissão Interamericana de Direitos Humanos, envolve diretamente o direito à vida e a segurança do paciente. O caso diz respeito à morte de dez recém-nascidos, em 1996, resultante de atos praticados por profissionais de saúde na Unidade de Tratamento Intensivo neonatal da referida clínica, conforme alegado pelos denunciantes. Ao todo, foram 82 recém-nascidos mortos, contudo somente os familiares de dez deles apresentaram petição, admitida em 2008 pela Comissão Interamericana de Direitos Humanos. De acordo com a petição apresentada junto à Comissão, os pacientes morreram em decorrência de infecção bacteriana adquirida no hospital devido a negligência médica, bem como à não observância de regras básicas de higiene - como usar luvas, lavar as mãos antes de tocar nos pacientes e trocar de vestimenta ou desinfetá-la previamente à adoção de procedimentos terapêuticos. Alguns familiares iniciaram uma investigação dos fatos e obtiveram provas da existência de vários surtos infecciosos na clínica referida. Apesar das denúncias acerca dos fatos relacionados à segurança do paciente apresentadas às autoridades estatais, os peticionários afirmaram que a clínica continuou internando crianças na UTI neonatal, sem terem sido adotadas medidas de segurança para erradicar as condições de antissepsia relatadas às autoridades estatais.

Quanto ao direito à vida dos pacientes, a Comissão assentou que estima pertinente levar em consideração que a morte das vítimas, em virtude da denunciada omissão do Estado no cumprimento do dever de fiscalizar a prestação dos serviços por parte da clínica mencionada, poderia caracterizar a violação do direito à vida, previsto no artigo 4.1 da Convenção Americana sobre Direitos Humanos ${ }^{30}$. Assim, por ocasião do relatório de mérito, a Comissão se comprometeu a analisar a obrigação do Estado de prevenir violações ao direito à vida, mediante a devida fiscalização dos serviços de saúde prestados por provedor privado e a investigação diligente acerca da morte dos pacientes ${ }^{31,32}$.

\footnotetext{
30"Artigo 4. Direito à vida - 1. Toda pessoa tem o direito de que se respeite sua vida. Esse direito deve ser protegido pela lei e, em geral, desde o momento da concepção. Ninguém pode ser privado da vida arbitrariamente." COMISSÃO INTERAMERICANA DE DIREITOS HUMANOS. Convenção Americana sobre Direitos Humanos. Disponível em: <https://www.cidh.oas.org/basicos/portugues/c.convencao_ americana.htm>. Acesso em: 07 set. 2016.

${ }^{31}$ COMISIÓN INTERAMERICANA DE DERECHOS HUMANOS. Report N. 70/08. Petición 12.242. Admisibilidad. Clínica Pediátrica de la Región de los Lagos. Brasil. 16 de octubre de 2008. Disponível em: <http://www. cidh.oas.org/annualrep/2008sp/Brasil12242.sp.htm>. Acesso em: 27 jan. 2015.

${ }^{32} \mathrm{O}$ Caso Laura Cornejo versus Equador diz respeito à alegação de morte da vítima em decorrência de má conduta profissional dos médicos que lhe deram uma dose mortal de morfina quando ela se encontrava internada em um hospital privado. Embora não seja uma situação propriamente de segurança do paciente, cabe assinalar que a Comissão Interamericana de Direitos Humanos não considerou a alegada violação do direito à vida da vítima, mas tão somente o dever do Estado de adotar as medidas legais cabíveis contra os médicos acusados de má conduta, ou seja, de assegurar o devido amparo legal e o acesso aos remédios judiciais adequados por parte dos peticionários no caso. Por outro lado, a Comissão entendeu que, mesmo tratando-se de profissionais sem qualquer vínculo com o Estado e tendo o fato ocorrido em um hospital privado, a responsabilidade internacional do Estado do Equador por violação de direitos humanos não pode ser afastada. Report n 69/02[1]. Admissibility. Petion 419/01. Laura Albán Cornejo. Ecuador. October 23, 2002.
} 
Cabe aos Estados, por meio de políticas, legislação e programas, a efetivação do direito à vida do paciente, obrigação essa que deriva dos tratados de direitos humanos e é monitorada pelos órgãos internacionais integrantes do Sistema ONU de Direitos Humanos e dos sistemas Europeu e Interamericano de Direitos Humanos. O direito à vida abarca o direito a cuidados seguros em saúde, ou seja, o paciente tem o direito de ter acesso com qualidade a tratamentos e a medicamentos, de modo que a intervenção terapêutica não lhe provoque danos ou morte. Para tanto, os agentes governamentais devem prevenir os danos decorrentes dos cuidados em saúde, de modo a materializar, no campo dos cuidados em saúde, a obrigação dos Estados de prevenir a violação de direitos humanos. Desse modo, a primeira obrigação estatal é a de evitar a ocorrência de mortes evitáveis, mormente quando o paciente se encontra em unidades de saúde com o objetivo de ser curado. Em seguida, passa-se ao exame da segurança do paciente relacionada à qualidade dos cuidados em saúde e, por consequência, integrante do direito à saúde.

\section{A segurança do paciente enquanto elemento do direito à saúde}

\section{Marco conceitual do direito à saúde}

$\mathrm{O}$ direito à saúde no plano internacional encontra-se previsto em diversos documentos, dentre eles, o artigo 12 do Pacto Internacional sobre os Direitos Econômicos, Sociais e Culturais ${ }^{33}$ é a maior referência normativa do citado direito. De acordo com o dispositivo mencionado, toda pessoa tem o direito de desfrutar o mais elevado nível possível de saúde física e mental - direito à saúde, que é definido pelo Comitê da ONU sobre os Direitos Econômicos, Sociais e Culturais como o direito ao desfrute de uma gama de bens, serviços e condições necessários para se alcançar o mais alto nível possível de saúde. O Comitê mencionado interpreta o direito à saúde não somente como o direito à atenção oportuna e apropriada à saúde, mas também aos principais fatores determinantes da saúde, como o acesso a água potável, condições sanitárias e de habitação adequadas, condições sadias de trabalho e meio ambiente, acesso a educação e informação sobre questões relacionadas à saúde (inclusive a saúde sexual e a reprodutiva). Ademais, o direito à saúde inclui serviços de promoção e prevenção no campo da saúde ${ }^{34}$.

Do ponto de vista teórico, o conteúdo do direito à saúde vem sendo desenvolvido pelos relatores especiais sobre o direito à saúde Paul Hunt e Anand Grover

\footnotetext{
33“ARTIGO 12. 1. Os Estados Partes do presente Pacto reconhecem o direito de toda pessoa de desfrutar o mais elevado nível possível de saúde física e mental." BRASIL. Decreto n. 591, de 6 de julho de 1992. Atos Internacionais. Pacto Internacional sobre Direitos Econômicos, Sociais e Culturais. Promulgação. Disponível em: <http://www.planalto.gov.br/ccivil_03/decreto/1990-1994/d0591.htm>. Acesso em: 07 set. 2016.

${ }^{34}$ COMITÉ SOBRE OS DIREITOS ECONÔMICOS, SOCIAIS E CULTURAIS. Observación general n. 14, 2000. Cuestiones sustantivas que se plantean en la aplicación del Pacto Internacional de Derechos Económicos, Sociales y Culturales. Disponível em: <http://www.acnur.org/t3/fileadmin/Documentos/BDL/2001/1451. pdf?view=1>. Acesso em: 23 jan. 2015.
} 
em relatórios produzidos entre os anos de 2003 a $2014^{35}$. Além disso, o marco referencial internacional do conteúdo do direito à saúde é o Comentário Geral n. 14/2002, elaborado pelo Comitê sobre Direitos Econômicos, Sociais e Culturais da ONU. Sendo assim, o direito à saúde conta com consolidado aporte teórico, que envolve o desenvolvimento dos elementos disponibilidade, acessibilidade, qualidade e aceitabilidade, bem como dos princípios da igualdade, accountability, participação, proteção dos vulneráveis e não discriminação.

Uma vez estabelecido que o direito à saúde não consiste no direito de estar saudável, mas sim no direito a um conjunto de bens, serviços e instalações - os quais devem ser disponíveis, acessíveis, aceitáveis e de qualidade -, pode-se indagar quais são as obrigações dos Estados. Consoante à tipologia obrigacional dos direitos humanos, os Estados têm o dever de respeitar, proteger e realizar o direito à saúde. Quanto à obrigação de respeitar, os Estados devem abster-se de negar ou limitar o acesso de todas as pessoas a bens e serviços de saúde, assim como de impor práticas discriminatórias; a obrigação de proteger implica atuar visando a que terceiros não violem o direito à saúde de seus jurisdicionados, por exemplo, estabelecendo requisitos para formação de profissionais de saúde e controlando a produção, distribuição e venda de medicamentos; por fim, a obrigação de realizar diz respeito à garantia da atenção à saúde, incluindo a promoção e a prevenção em saúde. Quanto à obrigação de realizar, indaga-se qual é a amplitude dessa obrigação, ou seja, qual é a cesta de bens e serviços que os Estados devem prover, direta ou indiretamente, na medida em que o direito à saúde é um direito de realização progressiva, pois depende de recursos orçamentários. Quanto a tal aspecto, embora se reconheçam as implicações financeiras concernentes à efetivação do direito à saúde, os Estados têm a obrigação de avançar constantemente o mais rápido e eficazmente até a plena realização do direito ${ }^{36}$.

Dessa forma, sob a perspectiva do direito à saúde, os Estados têm que assegurar a disponibilidade e a acessibilidade dos bens e serviços de saúde de qualidade, ética e culturalmente aceitáveis, bem como a acessibilidade a eles. Contudo, a jurisprudência internacional, a despeito de alguns parâmetros como os serviços de emergência $\mathrm{e}$ atenção primária à saúde, não assentam a cesta de bens e serviços que os Estados devem prover para todos. A Corte Europeia de Direitos Humanos decidiu que, quanto à obrigação positiva dos Estados de prover cuidados em saúde, pode ser configurada violação do direito à vida quando há a negativa do Estado de prover cuidados em saúde para um indivíduo em particular quando os mesmos

\footnotetext{
${ }^{35}$ UNITED NATION HUMAN RIGHTS. Office of the High Commissioner for Human Rights. Disponivel em: < http:// www.ohchr.org/EN/Issues/Health/Pages/AnnualReports.aspx>. Acesso em: 02 fev. 2015.

${ }^{36}$ COMITÉ SOBRE OS DIREITOS ECONÔMICOS, SOCIAIS E CULTURAIS. Observación general n. 14, 2000. Cuestiones sustantivas que se plantean en la aplicación del Pacto Internacional de Derechos Económicos, Sociales y Culturales, cit.
} 
cuidados se encontram disponíveis para a população em geral ${ }^{37}$. Nota-se que a Corte Europeia fez referência ao direito à vida, pois não julga violação direta ao direito à saúde, que se encontra previsto na Convenção Social Europeia ${ }^{38}$. Assim, a Corte vem apreciando casos relacionados ao direito à saúde por meio da análise da violação do direito à vida e do direito de não ser submetido a tortura, tratamento cruel, desumano ou degradante ${ }^{39}$. Na esfera da Comissão Europeia de Direitos Sociais, o acesso aos serviços de cuidados em saúde deve ser para toda a população; o gasto com bens e serviços de saúde por parte de pacientes integrantes de parcelas mais vulneráveis da sociedade há que ser minorado; o acesso aos tratamentos deve se dar com base em critérios transparentes, estabelecidos em nível nacional; e os custos dos serviços de saúde devem ser suportados, pelo menos em parte, pela comunidade como um todo $^{40}$. Assim, a discussão sobre a quais bens e serviços o paciente faz jus enquanto titular do direito à saúde diz respeito à política nacional dos Estados e, portanto, não é objeto desta investigação. Com efeito, a Corte Europeia de Direitos Humanos já deixou claro que, quando "a proteção contra doenças requerer o uso de recursos do Estado, os países são livres para determinar seus próprios objetivos e prioridades” ${ }^{41}$.

Como se viu, o direito à saúde compreende quatro elementos - disponibilidade, acessibilidade, qualidade e aceitabilidade -, assim como abarca os determinantes sociais da saúde. Tendo em conta a extensão do conteúdo do direito à saúde, optou-se por conferir ênfase a um de seus elementos, a qualidade. A qualidade dos cuidados em saúde impacta diretamente o gozo dos outros direitos humanos, como o direito à vida e o direito ao respeito pela vida privada, pois cuidados em saúde sem qualidade - como os providos por profissionais de saúde imperitos, baseados em tecnologia insegura ou não centrados no paciente - podem causar danos aos pacientes e desrespeitar seus valores e crenças privados. Sendo assim, em seguida será feita uma exposição introdutória sobre a qualidade na esfera dos cuidados em saúde para, em seguida, expor as contribuições teóricas sobre a qualidade enquanto elemento do direito à saúde, oriundas dos Relatores Especiais sobre o Direito à Saúde. Por fim, apresenta-se um caso julgado pela Corte Interamericana de Direitos Humanos concernente aos cuidados de qualidade em saúde.

\section{A qualidade como elemento do direito à saúde e a segurança do paciente}

Cuidados de baixa qualidade em saúde afetam diretamente os pacientes e elevam seus custos; por outro lado, a melhoria da qualidade previne que as pessoas

\footnotetext{
${ }^{37}$ ECHR. Case of Nitecki v. Poland. Admissibility. 21 March 2002.

${ }^{38} \mathrm{~A}$ competência substantiva da Corte cinge-se à Convenção Europeia dos Direitos Humanos.

${ }^{39}$ EUROPEANCOMMITTEEOFSOCIALRIGHTS. The right to healthand the European SocialCharter. Disponívelem: <http://www.coe.int/T/DGHL/Monitoring/SocialCharter/Theme\%20factsheets/FactsheetHealth_en.pdf> Acesso em: 20 fev. 2015.

${ }^{40}$ Id. Ibid.

${ }^{41}$ BRAZIER, M.; CAVE, E. Medicine, patients, and the law. 5. ed. London: Penguin Books, 2011. p. 39.
} 
busquem hospitais desnecessariamente ${ }^{42}$, bem como alivia a sensação de impotência e vulnerabilidade dos pacientes. A longa espera, a carência de informação, o desconforto das instalações e a insensibilidade em relação às necessidades dos pacientes repercutem negativamente em sua condição de saúde ${ }^{43}$. A qualidade nos cuidados em saúde é relevante para os pacientes, os profissionais de saúde, os provedores de serviços de saúde e para toda a população, porquanto auxilia a reduzir os custos e impacta o orçamento público e, por consequência, a renda familiar ${ }^{44}$.

A qualidade dos cuidados em saúde permanece sendo uma séria preocupação global. $\mathrm{O}$ aumento dos gastos em saúde ou do emprego de equipamentos médicos mais avançados não implica forçosamente o aperfeiçoamento em termos de qualidade. $\mathrm{O}$ envelhecimento populacional, acoplado às inovações médicas e à fragmentação dos sistemas de saúde, conduz ao aumento da importância da qualidade na área da saúde ${ }^{45}$. Os recursos gastos em saúde pelos países mais riscos foram duplicados nos últimos 30 anos, não gerando diretamente melhores resultados. No mesmo sentido, a ajuda financeira a países mais pobres com vistas ao atendimento das demandas sanitárias de suas populações, dentre elas a qualidade dos serviços de saúde, vem enfrentando persistentes desafios, tais como a fragmentação da coordenação dos serviços de saúde, os esforços paralelos e repetidos e a ausência de suporte técnico ${ }^{46}$.

Na literatura especializada sobre o tema, há certo consenso acerca da dificuldade de se definir o que seja qualidade em saúde ${ }^{47}$, pois ela é percebida de diferentes modos e se apresenta, enquanto propriedade dos cuidados em saúde, de variadas formas ${ }^{48}$. Reeves e Bednar assinalam que novas definições de qualidade em saúde são propugnadas sem rechaçar as que lhes antecedem. Assim, há uma ampla gama de concepções de qualidade empregadas atualmente ${ }^{49}$.

Donabedian, reconhecido precursor no campo da qualidade nos cuidados em saúde, assenta que ela é produto de dois fatores: um é a ciência e a tecnologia dos cuidados em saúde, e o outro consiste na aplicação da ciência e das tecnologias referidas na prática. Concretamente, a qualidade nos cuidados em saúde consiste no produto dos dois fatores assinalados, bem como se caracteriza por sete atributos, a saber: (a) eficácia, que é a capacidade da ciência e da tecnologia de produzir melhorias na saúde quando empregadas em circunstâncias favoráveis; (b) efetividade,

\footnotetext{
${ }^{42}$ ORGANISATION FOR ECONOMIC CO-OPERATION AND DEVELOPMENT - OECD. Forum on quality of care. Paris: OECD, 2010.

${ }^{43}$ MOULIN, Max. Delivering excellence in health and social care. Buckingham: Open, 2002.

${ }^{44}$ Id. Ibid.

${ }^{45}$ ORGANISATION FOR ECONOMIC CO-OPERATION AND DEVELOPMENT - OECD. Forum on quality of care, cit. ${ }^{46}$ WORLD HEALTH ORGANIZATION - WHO. Quality of care. Genebra: WHO, 2006.

${ }^{47}$ RANADE, W. A future for the NHS? Health Care for the Millennium. London: Longman, 1997.

${ }^{48}$ GONZÁLEZ RAMOS, Rosa María. La calidad de la atención en salud. Disponível em: <http://www. medigraphic.com/pdfs/oral/ora-2008/oras081a.pdf>. Acesso em: 20 fev. 2015.

${ }^{49}$ Apud MOULIN, Max. op. cit.
} 
que consiste no grau em que as melhorias na saúde são, de fato, realizadas; (c) eficiência, que é a habilidade de diminuir os custos dos cuidados em saúde sem afetar as melhorias alcançadas; (d) otimização, que significa o equilíbrio entre as melhorias dos cuidados em saúde e seu custo; (e) aceitabilidade, que é definida como a conformidade dos cuidados em saúde com os desejos e expectativas dos pacientes; (f) legitimidade, que exprime a conformidade com os padrões sociais, traduzidos em princípios éticos, leis e regulações em geral; e (g) equidade, que se constitui na adequação ao princípio que determina a distribuição equitativa de cuidados em saúde ${ }^{50}$.

De acordo com a OMS, a qualidade dos sistemas de saúde possui seis dimensões: (a) efetividade, os cuidados em saúde devem ser baseados em evidências científicas e prover melhorias na saúde dos indivíduos e comunidades; (b) eficiência, ofertar cuidados em saúde maximizando o uso dos recursos e evitando o desperdício; (c) acessibilidade, os cuidados em saúde devem ser oportunos, geograficamente acessíveis e serem oferecidos de modo que as habilidades e recursos sejam apropriados às necessidades médicas; (d) centralidade no paciente, o cuidado em saúde deve considerar as suas preferências e desejos individuais; (e) equidade, os cuidados em saúde não devem variar em qualidade em razão de gênero, raça, etnia e outros fatores pessoais; e (f) segurança, o cuidado em saúde deve ser efetivado de modo que os riscos sejam minimizados e os danos aos pacientes evitados ${ }^{51}$. Com base na categorização de Donabedian e da OMS, pode-se asseverar que, em linhas gerais, a qualidade em saúde sob a perspectiva do paciente implica resultados mais efetivos na melhoria de sua condição de saúde com base em suas necessidades, por meio do uso eficiente dos recursos humanos e materiais.

A qualidade nos cuidados em saúde pressupõe considerar a dinâmica relação entre estrutura, processo e resultado, que são os aspectos dos serviços de saúde. Estrutura refere-se aos recursos tangíveis, como instalações físicas, e intangíveis, como a conduta ética dos profissionais de saúde. O processo diz respeito às ações de cuidado, efetivadas com base em tais recursos, do ponto de vista clínico e não clínico. Os resultados decorrem dos recursos e das ações de cuidado em saúde e recaem sobre a população. A relação entre a qualidade e esses aspectos dos serviços de saúde apresenta-se sobremaneira complexa, descabendo abordá-la neste trabalho. Contudo, cabe salientar que o fato de se despenderem mais recursos financeiros não garante melhor qualidade, bem como em variadas situações inexiste consenso científico sobre qual é a "melhor prática" clínica, ou seja, a mais efetiva ${ }^{52}$. Por outro lado, aspectos não clínicos usualmente subestimados por profissionais e provedores de serviços de saúde - tais como ambientes limpos e aconchegantes, e a motivação dos profissionais de saúde -repercutem diretamente nos resultados em saúde.

\footnotetext{
${ }^{50}$ DONABEDIAN, Avedis. An introduction to quality assurance in Health care. Oxford: Oxford, 2003. ${ }^{51}$ WORLD HEALTH ORGANIZATION - WHO. Quality of care, cit.

${ }^{52}$ RANADE, W. op. cit.
} 
A efetivação da qualidade pressupõe o engajamento dos profissionais de saúde, dos pacientes e da comunidade ${ }^{53}$. Notadamente, o respeito ao paciente é peça crucial na qualidade dos cuidados em saúde, ou seja, os profissionais e provedores de saúde devem ter em conta os valores e as preferências dos pacientes, prover-lhes informação adequada e lhes garantir suporte emocional e conforto físico, pois sistemas de saúde alicerçados sobre as necessidades dos pacientes aprimoram os resultados em saúde e contribuem para sua eficácia ${ }^{54}$. A melhoria da qualidade dos serviços de saúde não depende necessariamente de amplas reformas nos sistemas de saúde dos países; dimensões da qualidade - como a segurança do paciente - podem ser aprimoradas sem profundas reformas ${ }^{55}$.

Os provedores de serviços de saúde devem assegurar que os serviços alcancem o mais alto padrão possível de qualidade e atendam às necessidades dos pacientes. As autoridades estatais dirigentes de serviços de saúde devem avaliar e comparar a qualidade dos serviços de saúde a fim de promover a accountability, de verificar o efetivo desenvolvimento das políticas de saúde e de auxiliar os provedores de serviço a aprender com as experiências de seus pares ${ }^{56}$.

Em suma, cuidados de qualidade em saúde caracterizam-se como aqueles prestados por profissionais de saúde altamente qualificados e que apresentam eficiência na utilização dos recursos, risco mínimo para os pacientes e resultados positivos em relação às condições de saúde dos pacientes ${ }^{57}$.

Nos relatórios produzidos pelos relatores especiais sobre direito à saúde, a qualidade dos bens, instalações e serviços de saúde (inclusive os cuidados) é enquadrada como um dos elementos do direito à saúde, em conjunto com a disponibilidade, a acessibilidade e a aceitabilidade. O desenvolvimento de sistemas de saúde efetivos e de boa qualidade é uma exigência decorrente do direito à saúde ${ }^{58}$. Nos relatórios é feita uma associação entre qualidade adequada e a segurança do paciente. Hunt, ao se referir à qualidade adequada, menciona o caso julgado pela Suprema Corte de Bangladesh sobre a importação de leite em pó contaminado com substância radioativa enquanto violação ao direito à saúde e à vida. No âmbito do caso citado, a Suprema Corte fixou a obrigação do Estado de melhorar da qualidade da saúde ${ }^{59}$. Hunt faz alusão ao problema dos medicamentos falsificados e contaminados ${ }^{60}$; dos medicamentos rejeitados nos países ricos por terem seu prazo de validade expirado

\footnotetext{
${ }^{53}$ WORLD HEALTH ORGANIZATION - WHO. Quality of care, cit..

${ }^{54}$ ORGANISATION FOR ECONOMIC CO-OPERATION AND DEVELOPMENT - OECD. Forum on quality of care, cit.

${ }^{55}$ WORLD HEALTH ORGANIZATION - WHO. Quality of care, cit.

${ }^{56}$ ORGANISATION FOR ECONOMIC CO-OPERATION AND DEVELOPMENT - OECD. Forum on quality of care, cit.

${ }^{57}$ GONZÁLEZ RAMOS, Rosa María. op. cit.

${ }^{58} \mathrm{HUNT}, \mathrm{P}$. Report to General Assembly (Main focus: Health systems and the right to the highest attainable standard of health). Disponivel em: <http://www.ohchr.org/EN/Issues/Health/Pages/AnnualReports. aspx>. Acesso em: 21 fev. 2015.

${ }^{59}$ Id. Ibid.

${ }^{60}$ ld. Ibid.
} 
ou serem inseguros e seu uso nos países pobres; e do sangue seguro ${ }^{61}$. Ademais, Hunt enfatiza que os Estados têm a obrigação de garantir a segurança, a eficácia e a qualidade dos medicamentos, bem como sua idoneidade e a precisão das informações correlatas a eles fornecidas para os profissionais de saúde e para a população ${ }^{62}$.

A qualidade adequada também demanda que os pacientes sejam tratados adequadamente - devendo os profissionais de saúde tratá-los com respeito e educação $0^{63}$ - e influencia a busca dos serviços de atenção à saúde por parte das mulheres ${ }^{64}$. Similarmente, Grover, ao abordar a temática da qualidade, alude à obrigação do Estado de proteger as populações dos medicamentos inseguros e de baixa qualidade, devendo regular seu registro e comércio, e à validade ética e médica das pesquisas biomédicas. Grover também se refere à informação sobre medicamentos, nos mesmos termos do exposto por Hunt ${ }^{65}$.

Na esfera da Corte Interamericana de Direitos Humanos, no Caso Suárez Peralta versus Equador a vítima foi internada para se submeter a cirurgia de apendicite, o que lhe provocou danos severos e permanentes. Na sentença sobre o caso, a Corte pontuou que, em 2012, a Assembleia Geral da OEA enfatizou a qualidade das instalações, bens e serviços de saúde, que exige a presença de pessoal treinado e condições médicas e sanitárias adequadas. De acordo com a Corte, para fins de cumprimento da obrigação de garantir o direito à integridade pessoal na esfera dos cuidados em saúde, os Estados devem contar com legislação apropriada para regular o provimento de serviços de saúde, estabelecendo normas de qualidade para os provedores de serviços de saúde públicos e privados, com vistas a impedir ou minorar os riscos de dano à integridade física dos pacientes.

A Corte assentou que os Estados devem contar com mecanismos de monitoramento e de controle dos serviços de saúde, bem como com procedimentos administrativos e judiciais destinados a apurar alegadas violações dos direitos humanos dos pacientes. Com efeito, os Estados têm o dever de prevenir os danos à integridade pessoal mediante o cumprimento de sua obrigação de regulação e fiscalização dos serviços de saúde. A Corte assentou que ${ }^{66}$ :

os Estados são responsáveis pela regulação [...] Prestação permanente de serviços e execução de programas nacionais objetivando serviços públicos de saúde de qualidade, de forma a desencorajar

\footnotetext{
${ }^{61}$ HUNT, P. Report to General Assembly (Main focus: Health systems and the right to the highest attainable standard of health), cit.

${ }^{62}$ Id. Ibid.

${ }^{63}$ Id. Ibid.

${ }^{64}$ Id. Ibid.

${ }^{65}$ GROVER, A. Report to General Assembly (Main focus: Access to medicines in the context of the right-tohealth framework). Disponivel em: <http://www.ohchr.org/EN/Issues/Health/Pages/AnnualReports. aspx>. Acesso em: 21 fev. 2015.

${ }^{66}$ CORTE INTERAMERICANA DE DIREITOS hUMANOS. Caso Suárez Peralta vs. Ecuador. Sentencia de 21 de mayo de 2013.
} 
qualquer ameaça ao direito à vida e à integridade física dos pacientes. Devem, particularmente, desenvolver os mecanismos adequados para inspecionar instituições [...] apresentar, investigar e resolver queixas e estabelecer procedimentos disciplinares ou judiciais adequados nos casos de má conduta profissional ou violação dos direitos dos pacientes ${ }^{67}$.

A obrigação do Estado de adotar medidas para salvaguardar a vida das pessoas sob sua jurisdição e garantir a qualidade dos cuidados em saúde impõe o fomento à formação e à capacitação apropriadas dos profissionais de saúde, bem como a adoção de mecanismos de monitoramento dos serviços de saúde visando à proteção da saúde e da vida dos pacientes ${ }^{68}$.

A qualidade dos cuidados em saúde é elemento essencial do direito à saúde, apresentando interface manifesta com o direito à vida do paciente. Nota-se que há uma discrepância entre o tratamento do tema da qualidade em saúde na literatura específica, na OMS e na jurisprudência internacional. Ou seja, nos órgãos de direitos humanos a questão da qualidade ainda não foi objeto de exame aprofundado. Na Relatoria sobre Direito à Saúde, constata-se que a qualidade vem sendo abordada primordialmente sob a perspectiva da segurança do paciente; já na Corte Interamericana de Direitos Humanos, a qualidade vem sendo associada ao dever do Estado de prevenir a ocorrência de dano ao paciente, particularmente o dano causado pela falha do Estado em fiscalizar os serviços de saúde e a atuação dos profissionais de saúde, lançando luz sobre a importância de sua formação e capacitação.

\section{Considerações Finais}

Atualmente, há evidência de que um número significativo de pacientes sofre danos decorrentes dos cuidados em saúde em hospitais, clínicas e similares, culminando, muitas vezes, em sua morte ${ }^{69}$. A segurança do paciente é um tema de direitos humanos, embora a comunidade internacional não lhe aplique uma abordagem baseada em tais direitos, mas tão somente a considere um problema de saúde pública. Reconhece-se que a própria identificação da segurança do paciente, enquanto elemento da qualidade, pelos organismos internacionais e órgãos nacionais de saúde consiste avanço notável na proteção dos direitos humanos dos pacientes, pois isso impele à adoção de medidas estatais que previnam a ocorrência de eventos adversos nos cuidados em saúde. Contudo, sustenta-se neste artigo que a aplicação do referencial teórico-normativo dos direitos humanos à segurança do paciente é relevante para a prevenção da ocorrência de danos ao paciente e, eventualmente,

\footnotetext{
${ }^{67}$ CORTE INTERAMERICANA DE DIREITOS HUMANOS. Caso Suárez Peralta vs. Ecuador, cit.

${ }^{68}$ Id. Ibid.

${ }^{69}$ WORLD HEALTH ORGANIZATION - WHO. The Multi-professional Patient Safety Curriculum Guide. Disponível em: <http://www.who.int/patientsafety/education/curriculum/en/>. Acesso em: 09 fev. 2015.
} 
sua morte. Isso porque os direitos humanos fundamentam-se na acepção de que os Estados têm a obrigação de prevenir a violação do direito do paciente à vida e à saúde, ensejando-lhe o dever de adotar medidas legislativas e administrativas com tal escopo. Sendo assim, na medida em que se advoga o dever dos Estados de prevenir a ocorrência de eventos adversos no processo terapêutico - impedindo, assim, o dano evitável ao paciente -, isso lhe confere o peso retórico e ético dos direitos humanos, enquanto linguagem internacionalmente compartilhada e mobilizadora de ativismo em prol da defesa dos direitos dos pacientes.

Constatou-se que órgãos internacionais de direitos humanos - tais como a Comissão Interamericana de Direitos Humanos, a Corte Europeia de Direitos Humanos e os relatores da ONU sobre direito à saúde - já analisaram casos concretos envolvendo a segurança do paciente sob a perspectiva dos direitos humanos. Dessas análises, pode-se constatar que a morte derivada de falhas sistêmicas ou individuais nos cuidados em saúde deve acarretar primordialmente a adoção de medidas que objetivem a prevenção do dano, tais como a fiscalização estatal dos serviços e bens de saúde com base em regras claras de segurança, e a formação e a capacitação de profissionais de saúde.

Por fim, à luz dos direitos humanos, o direito do paciente de não ter sua vida ceifada ou de sofrer danos resultantes dos cuidados em saúde, os quais supostamente existem para curá-lo, é derivado do direito à vida e do direito à saúde. Portanto, é imperioso que os Estados dediquem recursos humanos e financeiros com o fito de assegurar políticas públicas eficazes de segurança do paciente. Por outro lado, revela-se necessário para o enfrentamento dos eventos adversos na saúde que a sociedade civil se aproprie da temática da segurança do paciente, enquanto uma obrigação estatal, de modo a pressionar os governos com vistas à redução dos custos, humanos e financeiros e do cuidado em saúde inseguro para os indivíduos, a sociedade e o Estado.

\section{Referências}

ANNAS, George. The rights of patients. 3. ed. New York University Press, 2004.

ANVISA. Assistência segura: uma reflexão teórica aplicada à prática. Brasília: Anvisa, 2013.

BELETSKY, L.; EZER, T.; OVERALL, J.; BYRNE, I.; COHEN, J. Advancing human rights in patient care: the law in seven transitional countries. Disponível em: <http://www. opensocietyfoundations.org/sites/default/files/Advancing-Human-Rights-in-PatientCare-20130516.pdf>. Acesso em: 28 jan. 2015.

BRAZIER, M.; CAVE, E. Medicine, patients, and the law. 5. ed. London: Penguin Books, 2011.

COHEN, J.; EZER, T. Human rights in patient care: a theoretical and practical framework. Health and Human Rights, v.15, n. 2, p. 7-19, Dec. 2013. 
COMISIÓN DE DERECHOS HUMANOS. Recopilación de las Observaciones Generales Adoptadas por el Comité de Derechos Humanos de Naciones Unidas. No 6 - Derecho a la vida (art. 6). Disponível em: <http://www.derechos.org.ve/pw/wp-content/uploads/CDH1.pdf>. Acesso em: 8 fev. 2015.

COMISIÓN INTERAMERICANA DE DERECHOS HUMANOS. Report N. 70/08. Petición 12.242. Admisibilidad. Clínica Pediátrica de la Región de los Lagos. Brasil. 16 de octubre de 2008. Disponível em: <http://www.cidh.oas.org/annualrep/2008sp/Brasil12242.sp.htm>. Acesso em: 27 jan. 2015.

COMITÉ SOBRE OS DIREITOS ECONÔMICOS, SOCIAIS E CULTURAIS. Observación general n. 14, 2000. Cuestiones sustantivas que se plantean en la aplicación del Pacto Internacional de Derechos Económicos, Sociales y Culturales. Disponível em: $<$ www.un.org $>$. Acesso em: 23 jan. 2015.

CURRIE, L; WATT, S. An introduction to patient safety. In: CURRIE, Lynne (Ed.). Understanding patient safety. London: Quay Books, 2007. p. 11-26.

DONABEDIAN, A. An introduction to quality assurance in health care. Oxford: Oxford, 2003.

EUROPEAN COMMITTEE OF SOCIAL RIGHTS. The right to health and the European Social Charter. Disponível em: <http://www.coe.int/T/DGHL/Monitoring/SocialCharter/ Theme\%20factsheets/FactsheetHealth_en.pdf $>$. Acesso em: 20 fev. 2015.

FARIA, P.L.; MOREIRA, P.S.; PINTO, L.S. O direito e a segurança do paciente. In: SOUSA, Paulo; MENDES, W. Segurança do paciente: conhecendo os riscos nas organizações de saúde. Rio de Janeiro: FIOCRUZ, 2013. p. 115-134.

GONZÁLEZ RAMOS, Rosa María. La calidad de la atención en salud. Disponível em: <http:// www.medigraphic.com/pdfs/oral/ora-2008/oras081a.pdf>. Acesso em: 20 fev. 2015.

GROVER, A. Report to General Assembly (Main focus: Access to medicines in the context of the right-to-health framework). Disponível em: <http://www.ohchr.org/EN/Issues/Health/ Pages/AnnualReports.aspx>. Acesso em: 21 fev. 2015.

HERRING, J. Medical law. London: Pearson, 2013.

HUNT, P. Report to General Assembly (Main focus: Health systems and the right to the highest attainable standard of health). Disponível em: <http://www.ohchr.org/EN/Issues/Health/ Pages/AnnualReports.aspx>. Acesso em: 21 fev. 2015.

INSTITUTE OF MEDICINE. To err is human: building a safer health system. Washington: National Academy Press, 2000.

MENDES, Walter. Segurança do paciente. Disponível em: <http://portal.anvisa.gov.br/wps/ wcm/connect/8adff400417e01dd8ec6ee22d1e56fc9/Walter_Mendes.pdf?MOD=AJPERES>. Acesso em: 10 nov. 2015. 
MENDES, Walter. Taxonomia em segurança do paciente. In: SOUSA, Paulo; Mendes, Walter (Orgs.). Segurança do paciente: conhecendo os riscos nas organizações de saúde. Rio de Janeiro: FIOCRUZ, 2013.

MINISTÉRIO DA SAÚDE; FUNDAÇÃO OSWALDO CRUZ; AGÊNCIA NACIONAL DE VIGILÂNCIA SANITÁRIA. Documento de referência para o Programa Nacional de Segurança do Paciente. Disponível em: <http://bvsms.saude.gov.br/bvs/publicacoes/documento_ referencia_programa_nacional_seguranca.pdf>. Acesso em: 20 ago. 2015.

MOULIN, Max. Delivering excellence in health and social care. Buckingham: Open, 2002.

NATIONAL ACADEMY OF SCIENCES. Institute of Medicine: shaping the future for health. Disponível em: <https://www.iom.edu/ /media/Files/Report\%20Files/1999/To-Err-isHuman/To\%20Err\%20is\%20Human\%201999\%20\%20report\%20brief.pdf. Acesso em: 28 out. 2014.

ORGANISATION FOR ECONOMIC CO-OPERATION AND DEVELOPMENT - OECD. Forum on quality of care. Paris: OECD, 2010.

ORGANIZACIÓN MUNDIAL DE LA SALUD - OMS. 10 datos sobre seguridad del paciente. Disponível em: <http://www.who.int/features/factfiles/patient_safety/patient_safety_facts/ es/>. Acesso em: 30 jul. 2014.

Marco Conceptual de la Clasificación Internacional para la Seguridad del Paciente. Versión 1.1. 2009. Disponível em: <http://www.who.int/patientsafety/implementation/icps/ icps_full_report_es.pdf $>$. Acesso em: 28 out. 2014.

RANADE, W. A future for the NHS? Health Care for the Millennium. London: Longman, 1997.

REMON, C.A. OMS. Seguridad del paciente: curso virtual de introducción a la investigación en seguridad del paciente. OMS. Genebra: OMS, 2012. $480 \mathrm{~min}$. Disponível em: <http://www. who.int/patientsafety/research/curso_virtual/es/>. Acesso em: 6 out. 2016.

UNITED NATION HUMAN RIGHTS. Office of the High Commissioner for Human Rights. Disponível em: <http://www.ohchr.org/EN/Issues/Health/Pages/AnnualReports.aspx>. Acesso em: 02 fev. 2015.

WACHTER, Robert M. Personal accountability in healthcare: searching for the right balance. In: THE HEALTH Foundation, 2012. Traduzido pelo Proqualis - Instituto de Comunicação e Informação Científica e Tecnológica em Saúde; Fiocruz, 2013.

. Understanding patient safety. São Francisco: McGraw Hill, 2012.

WICKS, Elizabeth. Human rights and healthcare. London: Hart, 2007.

WORLD HEALTH ORGANIZATION - WHO. Quality of care. Genebra: WHO, 2006.

The Multi-professional Patient Safety Curriculum Guide. Disponível em: $<$ http://www. who.int/patientsafety/education/curriculum/en/>. Acesso em: 09 fev. 2015. 
A segurança do paciente à luz do referencial dos direitos humanos

Aline Albuquerque - Pós-Doutora em Direitos Humanos, University of Essex. Colchester, Inglaterra. Professora da Pós-Graduação em Bioética da Universidade de Brasília. Brasília/ DF, Brasil E-mail: alineaoliveira@hotmail.com. 